\title{
Ductal Eccrine Adenocarcinoma
}

National Cancer Institute

\section{Source}

National Cancer Institute. Ductal Eccrine Adenocarcinoma. NCI Thesaurus. Code C43345.

An adenocarcinoma that arises from eccrine glands. 